\title{
XMM-Newton observations of the first unidentified TeV gamma-ray source TeV J2032+4130^
}

\author{
D. Horns ${ }^{1}$, A. I. D. Hoffmann ${ }^{1}$, A. Santangelo ${ }^{1}$, F. A. Aharonian ${ }^{2}$, and G. P. Rowell ${ }^{3}$ \\ 1 Institute for Astronomy and Astrophysics Tübingen (IAAT) Sand 1, 72076 Tübingen, Germany \\ e-mail: horns@astro.uni-tuebingen.de \\ 2 Max-Planck Institut für Kernphysik (MPIK) PO Box 10 39 80, 69117 Heidelberg, Germany \\ 3 School of Chemistry and Physics, University of Adelaide, Australia
}

Received 29 November 2006 / Accepted 26 April 2007

\section{ABSTRACT}

\begin{abstract}
Context. The first unidentified very high energy gamma ray source (TeV J2032+4130) in the Cygnus region has been the subject of intensive search for a counterpart source at other wavelengths. In particular, observations in radio and X-rays are important to trace a population of non-thermal electrons.

Aims. A deep ( $\approx 50 \mathrm{ks}$ ) exposure of TeV J2032+4130 with XMM-Newton has been obtained. The large collection area and the field of view of the X-ray telescopes on-board of XMM-Newton allow to search for faint extended X-ray emission possibly linked to $\mathrm{TeV} \mathrm{J} 2032+4130$

Methods. The contribution of point sources to the observed X-ray emission from TeV J2032+4130 is subtracted from the data. The point-source subtracted X-ray data are analyzed using blank sky exposures and regions adjacent to the position of TeV J2032+4130 in the field of view covered by the XMM-Newton telescopes to search for diffuse X-ray emission.

Results. An extended X-ray emission region with a full width half maximum (FWHM) size of $\approx 12$ arcmin is found. The centroid of the emission is co-located with the position of $\mathrm{TeV} \mathrm{J} 2032+4130$. The angular extension of the X-ray emission region is slightly smaller than the angular size of $\mathrm{TeV} \mathrm{J} 2032+4130(F W H M=14 \pm 3 \mathrm{arcmin})$. The energy spectrum of the emission coinciding with the position and extension of $\mathrm{TeV} \mathrm{J} 2032+4130$ can be modeled by a power-law model with a photon index $\Gamma=1.5 \pm 0.2_{\text {stat }} \pm 0.3_{\text {sys }}$ and an energy flux integrated between 2 and $10 \mathrm{keV}$ of $f_{2-10 \mathrm{keV}} \approx 7 \times 10^{-13} \mathrm{erg} /\left(\mathrm{cm}^{2} \mathrm{~s}\right)$ which is lower than the very high energy gamma-ray flux observed from $\mathrm{TeV} \mathrm{J} 2032+4130$. The energy flux detected from the extended emission region is about a factor of two smaller than the summed contribution of the point sources present. The energy spectrum can also be fit with a thermal emission model from an ionized plasma with a temperature $k_{\mathrm{B}} T \approx 10 \mathrm{keV}$.

Conclusions. We conclude that the faint extended X-ray emission discovered in this observation is the X-ray counterpart of $\mathrm{TeV}$ J2032+4130. Formally, it can not be excluded that the extended emission is due to an unrelated population of faint, hot $\left(k_{\mathrm{B}} T \approx 10 \mathrm{keV}\right)$ unresolved point-sources which by chance coincides with the position and extension of $\mathrm{TeV} \mathrm{J} 2032+4130$. We discuss our findings in the frame of both hadronic and leptonic gamma-ray production scenarios.
\end{abstract}

Key words. acceleration of particles - gamma rays: observations - X-rays: general - gamma rays: theory

\section{Introduction}

Ground based air Cherenkov telescopes have detected in the last 6 years a number of unidentified sources of very high energy (VHE, $E>100 \mathrm{GeV}$ ) gamma-rays located in the Galactic plane. The first representative of these sources (TeV J2032+4130) was discovered serendipitously with the HEGRA telescope system in the Cygnus region (Rowell \& Horns 2002; Aharonian et al. 2002).

In a subsequent deep exposure for a total of $\approx 280 \mathrm{~h}$ of observation time the source detection was confirmed and its properties were studied in detail (Horns \& Rowell 2004; Aharonian et al. 2005a).

A remarkable feature of $\mathrm{TeV} \mathrm{J} 2032+4130$ is the fact that the angular extension of the source is $\left(6.2 \pm 1.2_{\text {stat }} \pm\right.$ $0.9_{\text {sys }}$ ) arcmin in radius which corresponds to a $F W H M=$ 14 arcmin. Gamma-ray emission from TeV J2032+4130 has

* Based on observations obtained with XMM-Newton, an ESA science mission with instruments and contributions directly funded by ESA Member States and NASA. been confirmed independently by the Whipple collaboration (Lang et al. 2004; Konopelko et al. 2006). A gamma-ray excess from the Cygnus region albeit more extended was recently reported by the Milagro collaboration (Abdo et al. 2006). The properties of $\mathrm{TeV} \mathrm{J} 2032+4130$ are common to most of the other unidentified VHE gamma-ray sources that have been discovered with the HESS telescopes so far (Aharonian et al. 2005b, 2006a): TeV J2032+4130 can be considered as the proto-type of the unidentified VHE gamma-ray sources known today.

So far, a number of point-like or moderately extended candidates for counterparts of TeV J2032+4130 have been identified at radio (Paredes et al. 2006; Butt et al. 2006b) and X-rays (Butt et al. 2003, 2006a; Mukherjee et al. 2003, 2006).

The origin and nature of TeV J2032+4130 remains unclear. It has been suggested that the nearby $(d=1.7 \mathrm{kpc})$ massive stellar OB association Cyg OB2 (see e.g. Knödlseder 2000) could be an accelerator of charged cosmic rays and consequently a site of gamma-ray production (Aharonian et al. 2002; Butt et al. 2006a; Aharonian et al. 2006b). This scenario has been strengthened by the recently discovered spatially extended 
VHE gamma-ray emission from the direction of the open stellar cluster Westerlund 2 (Aharonian et al. 2007).

Other possible explanations for the nature of $\mathrm{TeV} \mathbf{J} 2032+4130$ have been brought forward including gamma-ray production in possible jet lobes of Cyg X-3 (Aharonian et al. 2002), an unknown pulsar wind nebula (Bednarek 2003), or even extra-galactic source candidates as suggested by Mukherjee et al. (2003) and Butt et al. (2006b).

Independent of the nature of the source, two different gamma-ray production mechanisms are generally considered. In the hadronic scenario, gamma-rays are produced mainly via inelastic scattering of accelerated nuclei with the ambient medium (see e.g. Aharonian et al. 2005a; Reimer et al. 2006; Domingo-Santamaría \& Torres 2006). Alternatively, in the leptonic scenario gamma-rays are produced via inverse Compton scattering of ambient photons from a population of energetic electrons (Aharonian et al. 2005a; Reimer et al. 2006). Recently, it has been suggested, that excitation of giant dipole resonances of relativistic heavy nuclei in radiation dominated environments could be responsible for gamma-ray production in Cyg OB2 (Anchordoqui et al. 2006).

While the gamma-ray observations so far have been inconclusive with respect to the origin of the observed signal, X-ray observations can provide additional information to identify the origin of the emission and to discern between the two proposed scenarios.

In this Letter, we report the detection of spatially extended $\mathrm{X}$-ray emission coinciding with the position of $\mathrm{TeV} \mathrm{J} 2032+$ 4130 .

\section{Observations and data analyses}

The data were taken during two separate pointings of $X M M$-Newton. Table 1 summarizes the available data and configurations of the instruments used. The data were screened for soft proton flares following the method suggested in Read \& Ponman (2003). The detectors performed almost nominally: CCD \#6 of the MOS 1 camera had been switched off and CCD \#5 of MOS2 shows an increased instrumental background below $1 \mathrm{keV}$ which is two times larger than the background seen in the adjacent CCDs \#4 and \#6. However, above $1 \mathrm{keV}$ the background rate in CCD \#5 appeared consistent with the other detectors. The data suffer from contamination of single scattering events from the bright X-ray source Cyg X-3. The contamination is most prominent in the energy band above $5 \mathrm{keV}$. For this reason, the analyses that have been performed are constrained on the energy range from $1-5 \mathrm{keV}$ where the instrumental background and stray light contamination from Cyg X-3 are minimal. A closer inspection of the contamination using ray-tracing simulations has shown that the contribution of scattered light from Cyg X-3 to the X-ray emission seen from TeV J2032+4130 is negligible. At the same time, the vignetting of the telescopes is smallest in this energy range (Kirsch 2006; Pradas \& Kerp 2005).

Data reduction and analyses were performed using the Standard XMM-Newton Science Analysis Software SAS v7.0.

\subsection{Search for diffuse $X$-ray emission}

In an initial step of the data analyses, a catalogue of sources detected in the $1-5 \mathrm{keV}$ energy range is assembled using the edetect_chain task simultaneously for the MOS1, MOS2, and EPIC pn cameras for each of the two exposures. The minimum detection likelihood is set to a low value of 2 . This way,
$13.5 \%$ of the detected sources are due to statistical fluctuations while at the same time, more and fainter sources are detected than with the default value of 10 . A detailed study of the point sources detected in this XMM-Newton observation will be presented elsewhere. Here, the source catalogue has been used to generate model images of the point sources separately for each camera and pointing taking into account the position dependent point spread function of the XMM-Newton mirrors. These model images are then subtracted off the individual frames of the three cameras and for the two pointings. Within the $6.2^{\prime}$ region covered by the extension of the $\mathrm{TeV}$ source, a total energy flux of $f_{1-5 \mathrm{keV}} \approx 10^{-12} \mathrm{erg} /\left(\mathrm{cm}^{2} \mathrm{~s}\right)$ associated with point sources is observed. In order to subtract the particle and extragalactic photon background blank sky event files for the corresponding observation modes and filter settings have been obtained from the XMM guest observers' facility ${ }^{1}$. The blank field data have been processed to match the observational data and the resulting images are subtracted off the pointings towards $\mathrm{TeV} \mathbf{J} 2032+4130$. The images for the MOS1 and MOS2 cameras obtained from the two exposures are combined to form a background subtracted mosaic. The images of the EPIC pn camera have been omitted because of artefacts resulting from oversubtracting the contribution of point sources. For each of the four remaining images, exposure maps have been generated using the SAS task eexpmap. The exposure maps are combined in a mosaic which is then trimmed to a minimum exposure of $5 \mathrm{ks}$ corresponding to roughly $5 \%$ of the peak value. The background subtracted mosaic is then divided by the exposure map to obtain an exposure corrected, background subtracted, and source free image. Figure 1 shows three images of the combined exposures of the MOS1 and MOS2 cameras before the subtraction of the model images of the point sources (a), after the subtraction and smoothing with Gaussian of fixed width of 45 arcsec (b), and finally, the image after convolving it with a Gaussian of fixed width of 1.5 arcmin (c). Note the presence of scattered light along ring segments in the southern part of Fig. 1a. While Fig. 1b shows indications for the presence of a rim-like feature resembling the morphology observed in radio by Paredes et al. (2006), it should be noted that the subtraction of point-sources has an impact on the observed morphology on angular scales small compared to the extension of the detected extended emission. For a comparison with the extension and morphology of the gamma-ray source TeV J2032+4130, contours are overlaid to Fig. 1c. The X-ray image shows an extended emission region which is co-located with TeV J2032+4130 and of similar extension. The exposure corrected image of the diffuse emission is fit by a Gaussian with a width of $(5.1 \pm 0.3)$ arcmin which corresponds to a $F W H M=(11.7 \pm 0.6) \operatorname{arcmin}^{2}$.

\subsection{Energy spectrum of the diffuse $X$-ray emission}

In order to determine the energy spectrum of the diffuse X-ray emission, the contribution of the point sources has to be removed by excising regions around the sources. We use an excising region adapted to the point spread function and brightness of the source. The size of the region is chosen such that the relative contribution of the source to the local background is less than $20 \%$ at the boundary of the excised region. The wings of the point spread function lead to a contamination of the detected diffuse emission which amounts to $27 \%$ of the excess

\footnotetext{
1 ftp://xmm.esac.esa.int

2 All errors given throughout the paper are to the confidence of one standard deviation.
} 

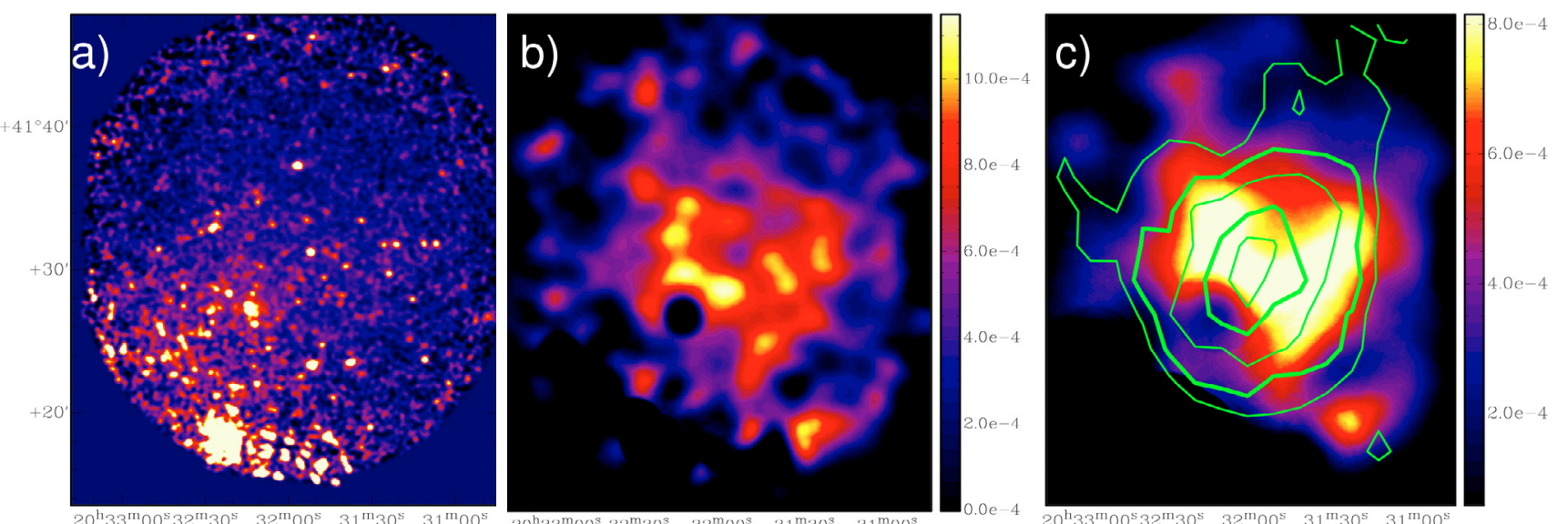

Fig. 1. Background subtracted and exposure corrected images obtained from the combined exposures of the MOS1 and MOS2 cameras (in units of counts/(s arcmin $\left.{ }^{2}\right)$. While the left image shows the emission including all point sources, the middle image shows the image after subtracting off all point sources, smoothed with a Gaussion of 45 arcsec width and with a changed scaling to highlight the extended X-ray emission present. Finally, the right image is obtained by smoothing the source subtracted image with a Gaussian of 1.5 arcmin width. The green contours indicate (in linear spacing) the significance contour of the HEGRA observations (starting at $3 \sigma$ ).

signal seen. This effect of contamination would be however compensated by the opposite effect caused by the diffuse emission present in the excised region $(\approx 25 \%)$ and therefore excluded from the reconstructed energy spectrum. We estimate the systematic uncertainty of this contamination to be $10 \%$ of the total flux. The energy spectra of a source region with a radius of $6.2^{\prime}$ centered on the position of TeV J2032+4130 from the MOS2 and EPIC pn cameras of the second pointing (ObsID 0305560201) are extracted. The second pointing is centered $5^{\prime}$ north of the centroid of $\mathrm{TeV} \mathrm{J} 2032+4130$ and therefore, we can choose a background region which is mirrored through the center of the field of view and has a similar acceptance as the source extraction region. Using the background region in the same field of view, the background estimate includes all relevant background components including X-ray emission from the Galactic ridge and the particle and instrumental background specific to this observation. The small azimuthal modulation of the acceptance of the MOS cameras can be neglected along this direction. The MOS1 camera is not used because the disabled CCD\#6 unfortunately does not allow to cover the entire source.

The response files are generated for these particular observations using the standard tools arfgen and rmfgen. Finally, the geometrical areas of the source and background regions are calculated taking gaps between the CCDs, bad pixels, and point source exclusion regions into account. The resulting background subtracted X-ray spectrum is fit with a power-law as well as an optically thin hot plasma model (apec) including photoelectric absorption (phabs). The spectral fitting of the two spectra was done using the xspec v11.3.2p spectral fitting package (Arnaud 1996). The fit describes the data well (see Table 2 for a summary of the fit parameters; only statistical $1 \sigma$ errors are quoted).

In order to estimate the influence of faint, unresolved point sources present in the stellar cluster, we have reduced X-ray imaging data taken with the Chandra satellite (Obs.\# 4501 for $48.6 \mathrm{ks}$ on $\mathrm{TeV} \mathrm{J} 2032+4130$ and Obs.\# 4511 for $98.7 \mathrm{ks}$ in the south-west of $\mathrm{TeV} \mathrm{J} 2032+4130$, centered on the core of Cyg OB2). Based upon a comparison of the Chandra and XMM-Newton source catalogues and the study of the $\mathrm{X}$-ray source population present in the Cyg OB2 cluster (Colombo et al. 2006), we estimate that point sources below the
Table 1. Summary of observation times and configurations on the target "TeV J2032+4130". All instruments were operated in full frame mode with a medium filter. The exposure quoted in parenthesis is for the EPIC pn camera.

\begin{tabular}{lcccc}
\hline \hline ObsID & Date & RA & Dec & Exposure \\
& & J2000 & J2000 & {$[\mathrm{ks}]$} \\
\hline 0305560101 & $2005-10-21$ & $20: 31: 57$ & $41: 29: 58$ & $27.3(23.6)$ \\
0305560201 & $2005-10-25$ & $20: 31: 57$ & $41: 34: 55$ & $25.8(20.5)$ \\
\hline
\end{tabular}

Table 2. Summary of the fit results of the X-ray energy spectrum with a power-law model (powerlaw) or thermal emission (apec with fixed solar abundances of the plasma) including photo-electric absorption (phabs).

\begin{tabular}{lrr}
\hline \hline Parameter & Value (powerlaw) & Value (apec) \\
\hline$N_{\mathrm{H}}\left[10^{21} \mathrm{~cm}^{-2}\right]$ & $3.5 \pm 1.6$ & $3.2 \pm 1.1$ \\
$\Gamma, k_{\mathrm{B}} T[\mathrm{keV}]$ & $1.5 \pm 0.2$ & $10.5 \pm 3.2$ \\
$f_{2-10 \mathrm{keV}\left[10^{-13} \mathrm{erg} /\left(\mathrm{cm}^{2} \mathrm{~s}\right)\right]}$ & $7.3 \pm 1.1$ & $7.4 \pm 1.1$ \\
$\chi_{\text {red }}^{2}$ (d.o.f.) & $1.02(862)$ & $1.01(861)$ \\
\hline
\end{tabular}

XMM-Newton detection limit can contribute up to $\approx 30 \%$ of the observed diffuse emission. It is however not straight-forward to correct the observed excess for the unresolved point sources detected with Chandra as the flux of the sources is generally found to vary with time. We estimate the systematic uncertainty on the flux conservatively to be $50 \%$ combining the influence of the unresolved point sources $(\approx 30 \%)$, uncertainties on the background subtraction $(\approx 10 \%)$, as well as the tails of the point spread functions of excised point sources $(\approx 10 \%)$. The total, unabsorbed energy flux derived from the model fit (see Table 2 ) is found to be $f_{2-10}=\left(7.3 \pm 3.5_{\text {sys }} \pm 1.1_{\text {stat }}\right) \times 10^{-13} \mathrm{erg} /\left(\mathrm{cm}^{2} \mathrm{~s}\right)$.

\section{Conclusions}

The XMM-Newton observations presented here indicate the presence of an extended ( $F W H M=11.7$ arcmin) X-ray source co-located with the first unidentified VHE gamma-ray source TeV J2032+4130 discovered with the HEGRA air Cherenkov telescope system. The size of the X-ray source is similar to the one of $\mathrm{TeV} \mathrm{J} 2032+4130$. The energy spectrum 


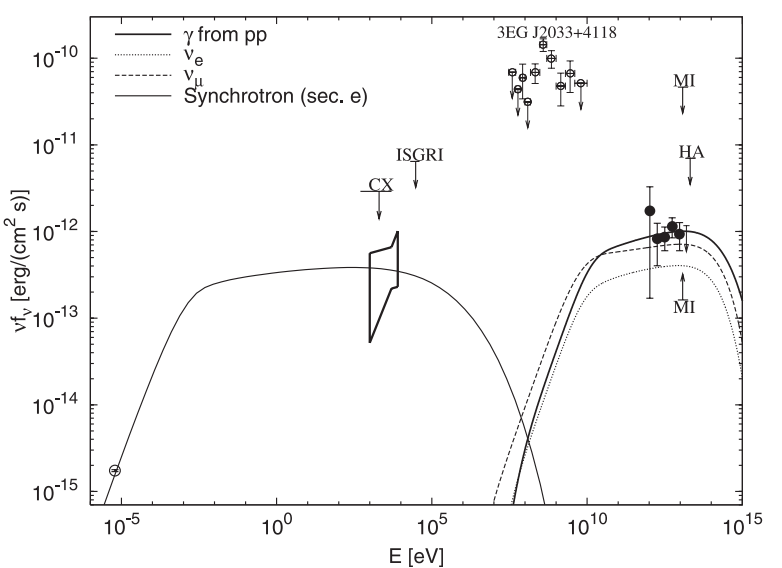

Fig. 2. The spectral energy distribution of TeV J2032+4130: the bowtie indicates the energy spectrum as measured with XMM-Newton including systematic and statistical uncertainties. The lines indicate the result of a model calculation for a hadronic gamma-ray and neutrino production scenario for a continuously active accelerator of protons up to a maximum energy of $5 \mathrm{PeV}$ at an age of $t_{\text {age }}=2500 \mathrm{yrs}$ and magnetic field $B=1 \mathrm{mG}$. The radio flux point of a possible nonthermal extended radio-source at $\lambda=20 \mathrm{~cm}$ (Paredes et al. 2006) is assumed to be an upper limit to the actual radio emission associated with TeV J2032+4130. The upper limits between $0.5-5 \mathrm{keV}$ (CX, Chandra) and 20-40 keV (ISGRI, INTEGRAL) are taken from Butt et al. (2006a). The energy spectrum of the EGRET source 3EG J2030+4118 (Hartman et al. 1999) is considered to be an upper limit. The Milagro (MI) upper limit is the integrated emission in a $3 \times 3\left({ }^{\circ}\right)^{2}$ region (Abdo et al. 2006) while the lower limit (MI) is scaled to the solid angle covered by TeV J2032+4130 assuming a uniform surface brightness. The upper limit from the HEGRA-AIROBICC (HA) wide angle Cherenkov detector is taken from Aharonian et al. (2002).

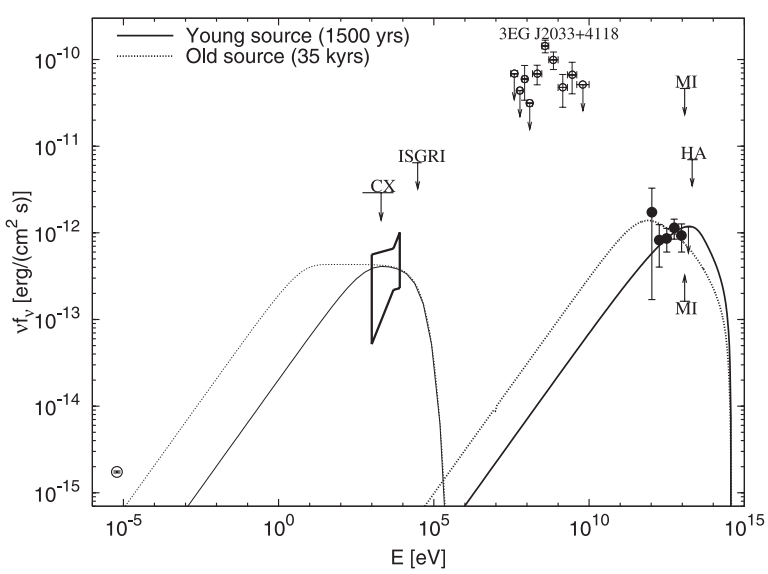

Fig. 3. In the leptonic scenario, the data are described well by a young source of an age of $1500 \mathrm{yrs}$, a magnetic field of $B=3 \mu \mathrm{G}$ and an energy density of the seed photon field of $w_{\mathrm{IR}}=3 \mathrm{eV} / \mathrm{cm}^{3}$ with a grey body temperature of $T=10 \mathrm{~K}$. As an alternative, an older source at an age of 35 kyrs is shown $\left(B=3 \mu \mathrm{G}, w_{\mathrm{IR}}=1 \mathrm{eV} / \mathrm{cm}^{3}\right)$. For a description of the multi-wavelength data see the caption of Fig. 2.

can be fit by a power-law model or by a thermal emission model with a plasma temperature of $k_{\mathrm{B}} T \approx 10 \mathrm{keV}$. The unabsorbed energy flux of the X-ray source in the energy range from $2-10 \mathrm{keV}$ is a factor of 2-3 smaller than the one observed from $\mathrm{TeV} \mathrm{J} 2032+4130$ at energies from 1-10 TeV.

We note that the observed extended X-ray emission could in principle be a so far unknown population of faint X-ray sources that are by chance distributed at an angular size which is similar to the one of TeV J2032+4130. The energy spectrum of these sources would have to be different from the bulk of the stellar X-ray sources detected from Cyg OB2 (Colombo et al. 2006) which on average show a thermal spectrum with $k_{\mathrm{B}} T \approx 1 \ldots 3 \mathrm{keV}$. Taking these considerations into account, we conclude that the observed extended X-ray emission is the X-ray counterpart of TeV J2032+4130.

Initial modeling of the X-ray and gamma-ray energy spectra using a hadronic gamma-ray production scenario indicates that the observed spectra can be naturally explained by a young (a few kyrs) "Pevatron" accelerator. The observation of synchrotron X-ray emission up to $\approx 5 \mathrm{keV}$ constrains the product of the square of maximum energy of accelerated protons $E_{\max }^{2}$ ) and magnetic field $(B)$ to exceed $E_{\max }^{2} \cdot B \gtrsim 5 \mathrm{PeV}^{2} \mathrm{mG}$. In this picture, the gamma-ray energy spectra would continue without cut-off well beyond $10 \mathrm{TeV}$. The hard X-ray emission from a similar source size region as the gamma-ray emission is a natural prediction within this model where the X-rays are produced by synchrotron emission of secondary electrons. Notably, in the frame of this model, the extended gamma-ray emission found by the Milagro collaboration would be explained by gamma-rays produced by accelerated particles of energies $\gtrsim 100 \mathrm{TeV}$ which have already escaped the accelerator.

A leptonic scenario provides a valid explanation of the observations as well (see Fig. 3). In this scenario, a young accelerator with a low magnetic field of a few $\mu \mathrm{G}$ accelerating electrons following a power-law distribution with $\mathrm{d} N / \mathrm{d} E \propto E^{-2}$ reaching up to energies of a few $100 \mathrm{TeV}$ can provide a good fit to the $\mathrm{X}$-ray and gamma-ray data. In contrary to the hadronic scenario, the gamma-ray energy spectrum above $10 \mathrm{TeV}$ is expected to be rather soft due to un-avoidable Klein-Nishina suppression of inverse Compton scattering. Furthermore, the seed photon density has to be higher than the average value in the interstellar medium (which is not unlikely given the possible proximity to the Cyg OB2 stellar association and the large stellar extinction towards that region indicating high density of dust). In the leptonic scenario, the X-ray spectrum can be expected to vary at different parts of the source due to cooling effects. More X-ray observations will be required to detect spectral variability.

Finally, neutrino observations will prove decisive to discern between the two emission scenarios (since neutrinos are only expected in the hadronic scenario). Even though the neutrino flux calculated here is very likely not detectable with the coming generation of neutrino telescopes like IceCube (Achterberg et al. 2006), it may be that the gamma-ray flux detected with Milagro from the same region is tracing the high energy particles which have already left the accelerator and fill a much larger volume. In this case, the region around $\mathrm{TeV} \mathrm{J} 2032+4130$ would be a powerful high energy neutrino source and would be detectable with future neutrino telescopes (Beacom \& Kistler 2007).

It should be noted that the observations of TeV J2032+4130 carried out with XMM-Newton are so far the deepest observations available for any of the unidentified VHE gamma-ray sources. It will be interesting to perform similar observations to search for faint, extended X-ray counterparts of other unidentified VHE gamma-ray sources.

Acknowledgements. We acknowledge the support of the Deutsches Zentrum für Luft- und Raumfahrt under grant number 50OR0302 and the support of the Eberhard Karls Universität Tübingen. We wish to thank Olaf Reimer and Facundo Albacete for providing the source lists found in the Chandra observations and Josep Paredes for making their results available prior to publication. This research has made use of NASA's Astrophysics Data System. We thank the anonymous referee for valuable comments. 


\section{References}

Abdo, A. A., Allen, B., Berley, D., et al. 2007, ApJ, 658, L33

Achterberg, A., et al. (Icecube Collaboration) 2006, Astropart. Phys., 26, 155

Aharonian, F., et al. (HEGRA coll.) 2002, A\&A, 393, L37

Aharonian, F., et al. (HEGRA coll.) 2005a, A\&A, 431, 197

Aharonian, F., et al. (HESS coll.) 2005b, Science, 307, 1938

Aharonian, F., et al. (HESS coll.) 2006a, ApJ, 636, 777

Aharonian, F., et al. (HEGRA coll.) 2006b, A\&A, 454, 775

Aharonian, F., et al. (HESS coll.) 2007, [arXiv: astro-ph/0703427]

Albacete Colombo, J. F., Flaccomio, E., Micela, G., Damiani, F., \& Sciortino, S. 2007, A\&A, 464, 211

Anchordoqui, L. A., Beacom, J. F., Goldberg, H., Palomares-Ruiz, S., \& Weiler, T. J. 2007, Phys. Rev. D, 75, 063001

Arnaud, K. A. 1996, Astronomical Data Analysis Software and Systems V, ASP Conf. Ser., 101, 17

Beacom, J. F., \& Kistler, M. D. 2007, Phys. Rev. D, submitted, [arXiv: astro-ph/0701751]

Bednarek, W. 2003, MNRAS, 345, 847

Butt, Y. M., Benaglia, P., Combi, J. A., et al. 2003, ApJ, 597, 494

Butt, Y. M., Drake, J., Benaglia, P., et al. 2006a, ApJ, 643, 238

Butt, Y. M., Combi, J. A., Drake, J., et al. 2006b, ApJ, submitted, [arXiv: astro-ph/0611731]
Domingo-Santamaría, E., \& Torres, D. F. 2006, A\&A, 448, 613

Hartman, R. C., Bertsch, D. L., Bloom, S. D., et al. 1999, ApJS, 123, 79

Horns, D., \& Rowell, G. 2004, New Astron. Rev., 48, 489

Horns, D., Aharonian, F., Santangelo, A., Hoffmann, A. I. D., \& Masterson, C. 2006, A\&A, 451, L51

Kelner, S. R., Aharonian, F. A., \& Bugayov, V. V. 2006, Phys. Rev. D, 74, 034018

Kirsch, M. 2006, in internal report XMM-SOC-CAL-TN-0018

Konopelko, A., et al. 2006, ApJ, accepted, [arXiv: astro-ph/0611730

Knödlseder, J. 2000, A\&A, 360, 539

Lang, M. J., Carter-Lewis, D. A., Fegan, D. J., et al. 2004, A\&A, 423, 415

Mukherjee, R., Halpern, J. P., Gotthelf, E. V., Eracleous, M., \& Mirabal, N. 2003, ApJ, 589, 487

Mukherjee, R., Gotthelf, E. V., \& Halpern, J. P. 2006,

[arXiv: astro-ph/0610299]

Paredes, J. M., Marti, J., Ishwara Chandra, C. H., \& Bosch-Ramon, V. 2007, ApJ, 654, 135

Pradas, J., \& Kerp, J. 2005, A\&A, 443, 721

Read, A. M., \& Ponman, T. J. 2003, A\&A, 409, 395

Reimer, A., Pohl, M., \& Reimer, O. 2006, ApJ, 644, 1118

Rowell, G., \& Horns, D. 2002, In Proceedings of The Gamma-Ray Universe, ed. A. Goldwurm, Les Arcs, France, 385

Waldron, W. L., Corcoran, M. F., Drake, S. A., \& Smale, A. P. 1998, ApJS, 118, 217 